\title{
Optimization of Sample Preparation Methods and SEM Imaging Conditions Enables High Resolution X-ray Mapping of Essential Elements in Biological Specimens
}

\author{
Louise Hughes $^{1}$, Patrick Trimby ${ }^{1}$ Joseph McKenna ${ }^{2}$ and Chris Hawes ${ }^{2}$ \\ 1. Oxford Instruments, High Wycombe, UK. \\ 2. Department of Biological and Medical Sciences, Oxford Brookes University, Oxford, UK.
}

Biological sample preparation is a major challenge for any type of electron microscopy. Energy dispersive X-ray spectrometry (EDS) detects the characteristic X-rays produced as a result of specimenbeam interactions in an electron microscope and identifies elements present in a sample. Scanning electron microscopy-EDS (SEM-EDS) is typically applied to hard materials using relatively high accelerating voltages and beam currents to produce accurate and quantitative data about sample composition. The application of SEM-EDS to biological samples has been limited in the past due to the fundamental nature of biological samples; they are extremely sensitive to the environment within an electron microscope and they are typically composed of lighter elements that can be difficult to analyze. Recent key developments in EDS technology [1] have increased the potential for accurate elemental analysis of biological samples, however, a major challenge remains in selecting the best methods for specimen preparation.

In general, samples such as cells and tissues require extensive fixation regimes to preserve structure, the addition of heavy metals for contrast generation and to reduce charging effects, dehydration and either drying for SEM, embedding in resin for transmission electron microscopy (TEM), or a combination of methods [2]. An alternative approach is cryo-fixation and cryo-electron microscopy, which provides preservation as close to the sample's living state as possible and is the fastest fixation method [2] but it is not always possible or suitable. A recent rise in the application of volume microscopy techniques such as array tomography, serial block face SEM and focused ion beam SEM have brought increasing demands on specimen preparation procedures $[3,4]$ and further widened the scope for the type of sample that could be analyzed with EDS.

We used plant (roots and leaves) and animal (liver, retina and cultured cells) samples prepared using standard SEM protocols as well as methods for resin embedded TEM samples. All samples were analyzed using EDS in an SEM. Standard SEM samples were fixed in $2.5 \%$ glutaraldehyde and $2 \%$ formaldehyde in $0.1 \mathrm{M}$ sodium cacodylate buffer $(\mathrm{pH} 7.4)$ for 1 hour at room temperature before being dehydrated in an ascending ethanol series, critical point dried, mounted onto aluminum stubs and coated in either carbon $(10 \mathrm{~nm})$, silver $(5 \mathrm{~nm})$, gold $(5 \mathrm{~nm})$ or left uncoated. Resin embedded samples were fixed as above and prepared using standard TEM protocols and either stained with ZIO for plant samples [4, 5], left unstained or stained en bloc with osmium and uranyl acetate prior to dehydration in ethanol, embedded in LR white or Epon 812 resin and mounted onto a stub as a block or sectioned onto either SEM stubs or glass slides. All samples were either coated as described above or left uncoated. EDS data was collected using the Ultim Max and Ultim Extreme detectors (Oxford Instruments, UK).

Resin-embedded samples were the most consistent in reducing common EDS artefacts generated by an uneven surface [6], however, they were more beam sensitive than standard SEM samples. Resin blocks were damaged by the beam to a greater extent than resin sections. Resin sections were the best samples for obtaining EDS spectra and maps of biological samples at low accelerating voltages. Uncoated 
sections (100nm thick) produced the best peak sensitivity and localization of elements in maps at $2.5 \mathrm{kV}$ (figure 1) with a clear differential distribution of elements such as oxygen, nitrogen and sodium. Carbon coated samples produced similar spectra to uncoated samples but with lower peak sensitivity. Localization of all elements in maps taken at the same pixel resolution and dwell time was worse than uncoated samples, probably due to the reabsorption of x-rays by carbon. Silver and gold (not shown) coated samples had the lowest peak sensitivity for elements at lower concentrations, however, the localization of elements that were detected, nitrogen and carbon, was better.

The optimization of biological sample preparation is a key aspect of EDS analysis. Care must be taken to select protocols that will ensure sample stabilization but without negatively impacting elemental analysis. Selection of the correct detector and imaging conditions for specimen type is also an important consideration.

References:

[1] A Janssen, M.G. Burke and S Burgess, In European Microscopy Congress 2016: Proceedings. (2016) p. 959.

[2] M Hayat in "Principles and Techniques of Electron Microscopy, Biological Applications",

(Cambridge University Press, Cambridge).

[3] CJ Peddie and LM Collinson, Micron, 61 (2014), p. 9.

[4] M Kittelmann, C Hawes and L Hughes, Journal of Microscopy 263 (2) (2016), p. 200.

[5] CR Hawes, B Juniper and JC Horn, Planta 152 (1981), p. 397.

[6] AV Girão, G Caputo and MC Ferro, in "Comprehensive Analytical Chemistry" 75 eds TAP RochaSantos and AC Duarte Elsevier, p. 153.
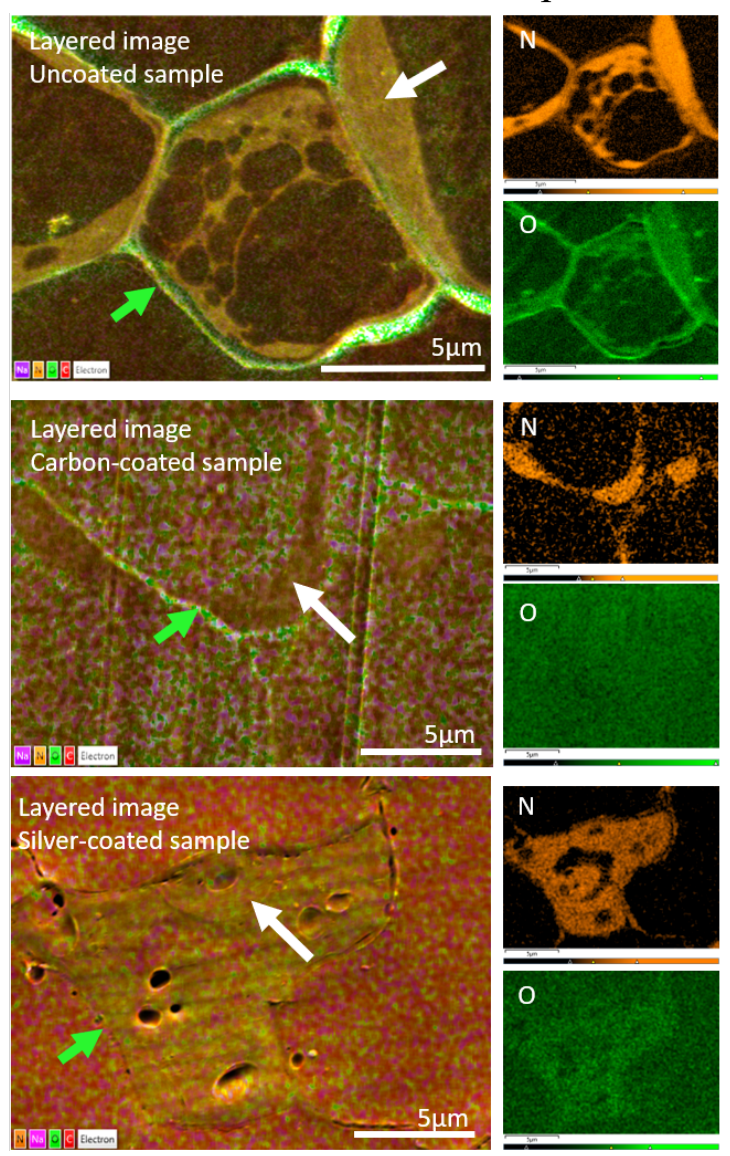
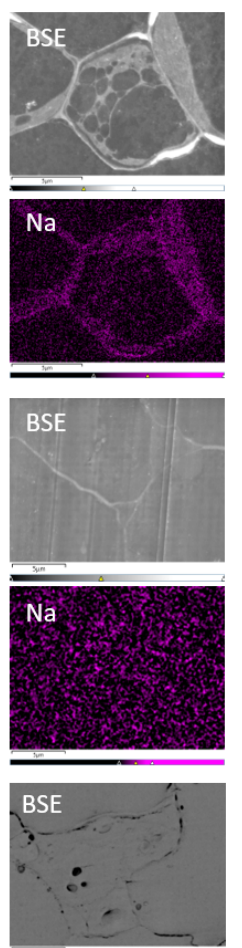

$\mathrm{Na}$
Figure 1. Layered EDS maps of uncoated (top), carbons coated (middle) and silver coated (bottom) sections of unstained Arabidopsis thaliana leaf tissue embedded in LR White resin with the corresponding maps for nitrogen $(\mathrm{N})$, oxygen $(\mathrm{O})$, sodium (Na) and the backscattered images (right). Chloroplasts (white arrows) and cell walls (green arrows) are indicated. 